Article

\title{
On the Growth of Some Functions Related to $z(n)$
}

\author{
Pavel Trojovský(i)
}

Department of Mathematics, Faculty of Science, University of Hradec Králové, 50003 Hradec Králové, Czech Republic; pavel.trojovsky@uhk.cz; Tel.: +42-049-333-2860

Received: 3 May 2020; Accepted: 26 May 2020; Published: 1 June 2020

Abstract: The order of appearance $z: \mathbb{Z}_{>0} \rightarrow \mathbb{Z}_{>0}$ is an arithmetic function related to the Fibonacci sequence $\left(F_{n}\right)_{n}$. This function is defined as the smallest positive integer solution of the congruence $F_{k} \equiv 0(\bmod n)$. In this paper, we shall provide lower and upper bounds for the functions $\sum_{n \leq x} z(n) / n, \sum_{p \leq x} z(p)$ and $\sum_{p^{r} \leq x} z\left(p^{r}\right)$.

Keywords: asymptotic; arithmetic functions; inequalities; Fibonacci sequence; order of appearance; Landau symbols

MSC: primary 111B39; secondary 11N37

\section{Introduction}

Perhaps the most important of the binary recurrences is the Fibonacci sequence $\left(F_{n}\right)_{n}$. This sequence starts with $F_{0}=0$ and $F_{1}=1$ and it satisfies the 2nd order recurrence relation $F_{n+2}=$ $F_{n+1}+F_{n}$ (for $n \geq 0$ ). A well-known, explicit, formula for the $n$th Fibonacci number is called the Binet-formula

$$
F_{n}=\frac{\alpha^{n}-\beta^{n}}{\sqrt{5}},
$$

where $\alpha:=(1+\sqrt{5}) / 2$ and $\beta:=(1-\sqrt{5}) / 2$. It follows from this formula that the estimates $\alpha^{n-2} \leq F_{n} \leq \alpha^{n-1}$, hold for all $n \geq 1$.

The study of the divisibility properties of Fibonacci numbers has always been a popular area of research. For example, it is still an open problem to decide if there are infinitely many primes in that sequence. In order to study such kind of Diophantine problems, the arithmetic function $z: \mathbb{Z}_{>0} \rightarrow \mathbb{Z}_{>0}$ was defined by setting $z(n)=\min \left\{k \geq 1: n \mid F_{k}\right\}$. This function is called the order of appearance in the Fibonacci sequence. For more results on $z(n)$, see [1] and references therein.

In 1878, Lucas ([2], p. 300) established that $z(n)$ is well defined and, in 1975, J. Sallé [3] proved that $z(n) \leq 2 n$, for all positive integers $n$. This is the sharpest upper bound for $z(n)$, since for example

$$
z(n)=2 n \quad \text { if and only if } n=6 \cdot 5^{k}, \text { for } k \geq 0 .
$$

However, apart from these cases this upper bound is very weak. For instance, $z(2255)=20<$ $10^{-2} \cdot 2255$. In fact, Marques [4] gave sharper upper bounds for $z(n)$ for all positive integers $n \neq 6 \cdot 5^{k}$. These upper bounds depend on the number of distinct prime factors of $n$, denoted by $\omega(n)$.

In the main stream of the Analytic Number Theory, we have the three following functions

$$
H(x):=\sum_{n \leq x} \frac{1}{n}, \vartheta(x):=\sum_{p \leq x} \log p \text { and } \psi(x):=\sum_{n \leq x} \Lambda(n),
$$

where $\Lambda(n)$ is the well-known von Mangoldt function defined as $\log p$ if $n=p^{r}$, for some prime number $p$ and $r \geq 1$, and 0 otherwise (see, e.g., $[5,6]$ ). The functions $\vartheta(x)$ and $\psi(x)$ are called the first 
and the second Chebyshev functions, respectively. Note that $\psi(x)$ can be rewritten as $\sum_{p^{r} \leq x} \log p$. Here (and in all what follows) $\sum_{n \leq x}, \sum_{p \leq x}$ and $\sum_{p^{r} \leq x}$ mean that the sum is taken over all positive integers, all prime numbers and all prime powers belonging to the interval $[1, x]$, respectively.

Probably, the main importance of the functions $\psi$ and $\vartheta$ relies in the proof of the celebrated Prime Number Theorem which states that

$$
\pi(x) \sim \frac{x}{\log x}
$$

where $\pi(x)=\sum_{p \leq x} 1$ is the prime counting function. Indeed, the prime number theorem and the statements $\vartheta(x) \sim x$ and $\psi(x) \sim x$ are all equivalent. Here $f(x) \sim g(x)$ (asymptotic equivalence) means that $f(x) / g(x)$ tends to 1 as $x \rightarrow \infty$ (in another way, $f(x)=g(x)+o(g(x))$ where $o(g(x))$ means a function $h(x)$ with $\lim _{x \rightarrow \infty} h(x) / g(x)=0$ ). Actually, one has the following stronger fact

$$
\pi(x)=\frac{x}{\log x}+O\left(\frac{x}{(\log x)^{2}}\right) .
$$

Here we shall use the Landau symbols in their usual meaning, i.e., we say that $f(x)=O(g(x))$ (or $f \ll g$ ), if there exists a positive constant $M$ such that $|f(x)| \leq M|g(x)|$, for all sufficiently large $x$. Also, $f \asymp g$ means that $f \ll g$ and $g \ll f$.

Another function of great interest is the harmonic function $H(x)$ whose image for $x \in \mathbb{Z}_{>0}$ is called the $x$ th harmonic number and denoted by $H_{x}$. These numbers gained much attention with their relation to the Riemann hypothesis. In fact, the Riemann hypothesis is equivalent to prove that

$$
d(n) \leq H_{n}+e^{H_{n}} \log H_{n}
$$

for all $n \geq 1$, where $d(n)$ is the sum of the positive divisors of $n$ (see [7]). We observe that the harmonic series, i.e., $\lim _{x \rightarrow \infty} H(x)$ is a well-studied example of divergent series. In fact, it holds that

$$
H(x)=\log x+O(1)
$$

which agrees with its very slow divergence.

In this paper, we are interested in studying the growth of the following Fibonacci versions of $H(x), \vartheta(x)$ and $\psi(x)$, thus, the functions $Z_{H}(x), Z_{\vartheta}(x)$ and $Z_{\psi}(x)$ (see Figure 1 ), for a positive real $x$, which are defined as

$$
Z_{H}(x):=\sum_{n \leq x} \frac{z(n)}{n}, Z_{\vartheta}(x):=\sum_{p \leq x} z(p) \text { and } Z_{\psi}(x):=\sum_{p^{r} \leq x} z\left(p^{r}\right) .
$$

First, observe that since $1 \leq z(n) \leq 2 n$, then the following trivial estimates hold

$$
\log x \ll H(x) \leq Z_{H}(x) \leq \sum_{n \leq x} 2 \leq 2 x .
$$

However, we found the previous bounds by neglecting the contribution of $z(n)$ (which is much bigger than 1 and much smaller than $2 n$, in almost all cases). In fact, by taking $z(n)$ into account, we obtain

Theorem 1. We have that

$$
(\log x)^{2} \ll Z_{H}(x) \ll \frac{x}{(\log x)^{1 / 3}} .
$$

For the function $Z_{\vartheta}(x)$, if we use that $z(p) \geq 1$, we get

$$
Z_{\vartheta}(x) \geq \sum_{p \leq x} 1=\pi(x) \gg \frac{x}{\log x} .
$$


Again, with an extra effort, we can improve this by proving that

Theorem 2. We have that

$$
x \ll Z_{\vartheta}(x) \ll \frac{x^{2}}{\log x} .
$$

Since the number of prime powers in $[1, x]$ is bigger than $\pi(x)$, a similar direct inequality (that one for $Z_{\vartheta}(x)$ ) could be derived for $Z_{\psi}(x)$. However, by using the behavior of $z\left(p^{r}\right)$, we can obtain better estimates such as

Theorem 3. We have that

$$
x \ll Z_{\psi}(x) \ll \frac{x^{2}}{\log x} .
$$

Note that even with a larger number of possibilities in the sum of $Z_{\psi}(x)$, its bounds are the same (in order) than the ones for $Z_{\vartheta}(x)$ (Theorem 2). The explanation for this, follows from the fact that the contribution, i.e., the number of powers of $p$ (for example) belonging to $[1, x]$ is $O(\log x)$ which is $o(x)$. In other words, this amount is almost negligible (compared with $x$, in terms of order).

In a few words, the proof of the results combine some new (sharper upper bounds for $z(n)$ due to Marques) and classical results (such as results due Abel, Sathé, Selberg) in Number Theory.

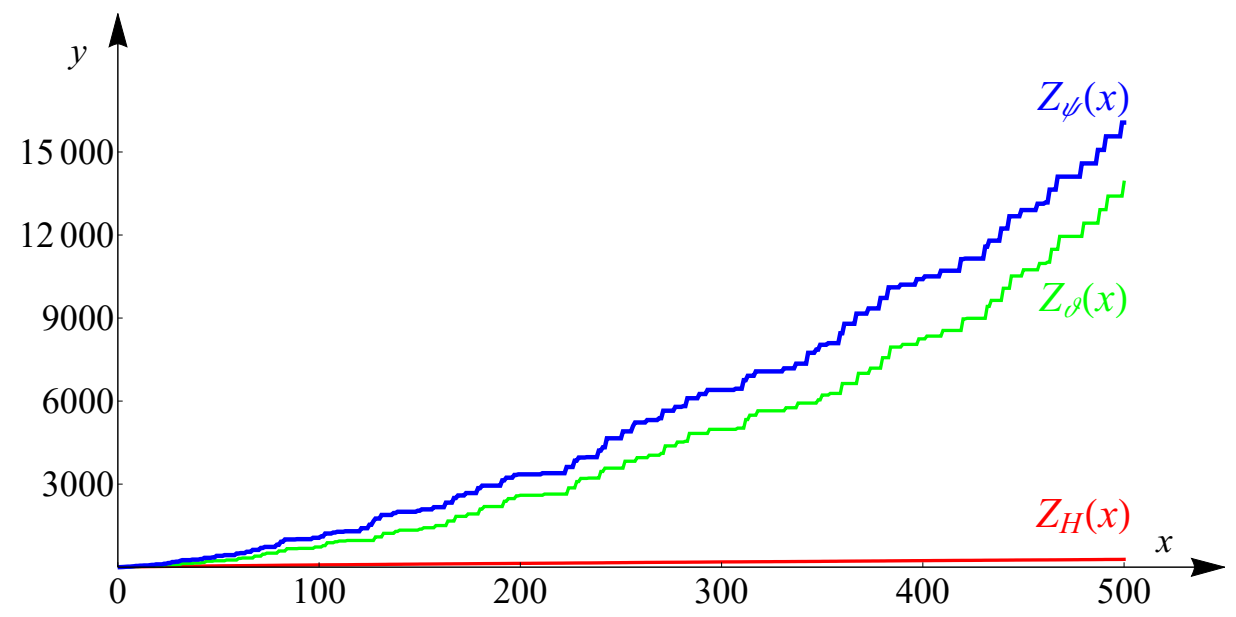

Figure 1. The graphs of the functions $Z_{H}(x), Z_{\vartheta}(x)$ and $Z_{\psi}(x)$ for $x \in(0,500]$.

\section{Auxiliary Results}

In this section, we shall present some tools which will be very useful in the proofs. We start with some results due to Marques [4], which will be very helpful in our proof. Thus, we shall state his results as lemmas (in what follows, the 2-adic valuation of $n$ is $v_{2}(n)=\max \left\{k \geq 0: 2^{k} \mid n\right\}$ ).

Lemma 1. We have

(i) $z\left(2^{k}\right)=3 \cdot 2^{k-2}($ for $k \geq 3), z\left(3^{k}\right)=4 \cdot 3^{k-1}$ (for $k \geq 1$ ) and $z\left(5^{k}\right)=5^{k}($ for $k \geq 0)$.

(ii) If $p>5$ is a prime, then

$$
z\left(p^{k}\right) \leq\left(p-\left(\frac{5}{p}\right)\right) p^{k-1}, \text { for } k \geq 1
$$

where, as usual, $\left(\frac{a}{q}\right)$ denotes the Legendre symbol of a with respect to a prime $q>2$.

Lemma 2. Let $n$ be an odd integer number with $\omega(n) \geq 2$, then

$$
z(n) \leq 2 \cdot\left(\frac{2}{3}\right)^{\omega(n)-\delta_{n}} n
$$


where

$$
\delta_{n}= \begin{cases}0, & \text { if } 5 \nmid n ; \\ 1, & \text { if } 5 \mid n\end{cases}
$$

Lemma 3. Let $n$ be an even integer number with $\omega(n) \geq 2$, it holds that

(i) If $v_{2}(n) \geq 4$, then

$$
z(n) \leq \frac{3}{4} \cdot\left(\frac{2}{3}\right)^{\omega(n)-\delta_{n}-1} n
$$

(ii) If $v_{2}(n)=1$, then

$$
z(n) \leq \begin{cases}3 n / 2, & \text { if } \omega(n)=2 \text { and } 5 \mid n ; \\ 2 n, & \text { if } \omega(n)=2 \text { and } 5 \nmid n ; \\ 3 \cdot(2 / 3)^{\omega(n)-\delta_{n}-1} n, & \text { if } \omega(n)>2 .\end{cases}
$$

(iii) If $v_{2}(n) \in\{2,3\}$, then

$$
z(n) \leq \begin{cases}3 n / 2, & \text { if } \omega(n)=2 \text { and } 5 \mid n ; \\ n, & \text { if } \omega(n)=2 \text { and } 5 \nmid n ; \\ (2 / 3)^{\omega(n)-\delta_{n}-2} n, & \text { if } \omega(n)>2 .\end{cases}
$$

The next lemma is a powerful result in analytic number theory which is related to positive integers with fixed number of distinct prime factors.

Lemma 4 (Sathé-Selberg Formula). For any positive constant $A$, we have

$$
\# \mathcal{P}_{k}(x):=\#\{n \leq x: \omega(n)=k\} \sim G\left(\frac{k-1}{\log \log x}\right) \frac{x}{\log x} \frac{(\log \log x)^{k-1}}{(k-1) !},
$$

for $x \geq 3$ and $1 \leq k \leq A \log \log x$, where

$$
G(z):=\frac{1}{\Gamma(1+z)} \prod_{\text {p prime }}\left(1+\frac{z}{p}\right)\left(1-\frac{1}{p}\right)^{z} .
$$

In the previous statement $\Gamma(z)=\int_{0}^{\infty} x^{z-1} e^{-x} d x$ (for $x>0$ ) is the well-known Gamma function. The proof of Lemma 4 can be found in $[8,9]$.

Our last tool is a very useful formula due to Abel which makes an interplay between a discrete sum and an integral (continuous sum). More precisely,

Lemma 5 (Abel's Summation Formula). Let $\left(a_{n}\right)_{n}$ be a sequence of real numbers and define its partial sum $A(x):=\sum_{n \leq x} a_{n}$. For a real number $x>1$, let $f$ be a continuously differentiable function on $[1, x]$. Then

$$
\sum_{n \leq x} a_{n} f(n)=A(x) f(x)-\int_{1}^{x} A(t) f^{\prime}(t) d t .
$$

Remark 1. We remark that, throughout what follows, the implied constants in $\ll$ and $\gg$ can be made explicit. Here, we decided to use asymptotic bounds in order to leave the text more readable. However, we shall provide the explicit inequalities for convenience of the reader (they can be found in [10], for example).

(i) $\sum_{n \leq x} \frac{1}{n}>\log (x+1)$; 
(ii) $\vartheta(x)<1.000028 x$, for $x>0$ and $\vartheta(x)>0.998684 x$, for $x>1319007$;

(iii) $p_{n}<n \log n+n \log \log n$, for $n \geq 6$;

(iv) $\frac{x}{\log x}<\pi(x)<1.25506 \frac{x}{\log x}$, for $x \geq 17$.

As usual, from now on we use the well-known notation $[a, b]=\{a, a+1, \ldots, b-1, b\}$, for integers $a<b$.

Now we are ready to deal with the proof of our results.

\section{The Proofs}

3.1. The Proof of Theorem 1

Since, by definition, $n \mid F_{z(n)}$, then $n \leq F_{z(n)} \leq \alpha^{z(n)-1}$ and $\operatorname{so} z(n)>\log n / \log \alpha$. Thus

$$
Z_{H}(x)=\sum_{n \leq x} \frac{z(n)}{n} \gg \sum_{n \leq x} \frac{\log n}{n} .
$$

Now, we shall use Lemma 5 for $a_{n}=1 / n$ and $f(x)=\log x$. Then

$$
\sum_{n \leq x} \frac{\log n}{n}=H(x) \log x-\int_{1}^{x} \frac{H(t)}{t} d t
$$

Since $H(x)=\log x+O(1)$ and $\int_{1}^{x} \frac{\log t}{t} d t=(\log x)^{2} / 2$, then

$$
\begin{aligned}
\sum_{n \leq x} \frac{\log n}{n} & =(\log x+O(1)) \log x-\int_{1}^{x} \frac{\log t+O(1)}{t} d t \\
& =\frac{1}{2}(\log x)^{2}+O(\log x) \gg(\log x)^{2}
\end{aligned}
$$

and so $Z_{H}(x) \gg(\log x)^{2}$.

For the second part, we use Lemmas 1,2 and 3 to derive that

$$
z(n) \leq 7\left(\frac{2}{3}\right)^{\omega(n)} n
$$

for all $n>1$. First, let us write $Z_{H}(x)$ as

$$
Z_{H}(x)=\sum_{k=1}^{h(x)} \sum_{n \in \mathcal{P}_{k}(x)} \frac{z(n)}{n}
$$

where $h(x)=\max \{\omega(t): t \leq x\}$. By using that $z(n) \leq 7 \cdot(2 / 3)^{\omega(n)} n$, we have

$$
Z_{H}(x) \ll \sum_{k=1}^{h(x)} \sum_{n \in \mathcal{P}_{k}(x)}\left(\frac{2}{3}\right)^{\omega(n)} \ll \sum_{k=1}^{h(x)}\left(\frac{2}{3}\right)^{k} \# \mathcal{P}_{k}(x)
$$

which can be written as

$$
Z_{H}(x) \ll \sum_{k=1}^{\lfloor\log \log x\rfloor}\left(\frac{2}{3}\right)^{k} \# \mathcal{P}_{k}(x)+\sum_{k \geq\lfloor\log \log x\rfloor+1}\left(\frac{2}{3}\right)^{k} \# \mathcal{P}_{k}(x)
$$


Now, we shall use Lemma 4 to deal with the first sum in the right hand side above. Since $G(z)$ converges uniformly and absolutely in any bounded set, we have $\max _{z \in[0,1]}\{|G(z)|\} \leq C$, for some positive constant $C$. Now, by Lemma 4 for $A=1$, we get $\left|G\left(z_{k}\right)\right| \leq C\left(\right.$ for $\left.z_{k}:=(k-1) / \log \log x<1\right)$ and

$$
\begin{aligned}
\sum_{k=1}^{\lfloor\log \log x\rfloor}\left(\frac{2}{3}\right)^{k} \# \mathcal{P}_{k}(x) & \ll \sum_{k=1}^{\lfloor\log \log x\rfloor}\left(\frac{2}{3}\right)^{k} G\left(z_{k}\right) \cdot \frac{x}{\log x} \frac{(\log \log x)^{k-1}}{(k-1) !} \\
& \ll \frac{x}{\log x} \sum_{k \geq 1} \frac{\left(\log (\log x)^{2 / 3}\right)^{k-1}}{(k-1) !} \\
& \ll \frac{x}{\log x} \exp \left(\log (\log x)^{2 / 3}\right)=\frac{x}{(\log x)^{1 / 3}} .
\end{aligned}
$$

Therefore,

$$
\sum_{k=1}^{\lfloor\log \log x\rfloor}\left(\frac{2}{3}\right)^{k} \# \mathcal{P}_{k}(x) \ll \frac{x}{(\log x)^{1 / 3}} .
$$

For the second sum in the right hand side of (6), we use that $\# \mathcal{P}_{k}(x) \leq x$ to obtain

$$
\sum_{k \geq\lfloor\log \log x\rfloor+1}\left(\frac{2}{3}\right)^{k} \# \mathcal{P}_{k}(x) \ll\left(\frac{2}{3}\right)^{\log \log x} x,
$$

where we used that $\lfloor\log \log x\rfloor+1>\log \log x$. Since $3 / 2>\sqrt[3]{e}$, then

$$
\left(\frac{3}{2}\right)^{\log \log x}>e^{(\log \log x) / 3}=(\log x)^{1 / 3} .
$$

Thus

$$
\sum_{k \geq\lfloor\log \log x\rfloor+1}\left(\frac{2}{3}\right)^{k} \# \mathcal{P}_{k}(x) \ll \frac{x}{(\log x)^{1 / 3}} .
$$

By combining (6), (7) and (8), we obtain the desired result.

\subsection{The Proof of Theorem 2}

By the Prime Number Theorem, we have that $\vartheta(x) \sim x$. In particular, it holds that $\vartheta(x) \gg x$. Since $\vartheta(x)=\sum_{p \leq x} \log p$, then

$$
Z_{\vartheta}(x)=\sum_{p \leq x} z(p) \gg \sum_{p \leq x} \log p=\vartheta(x) \gg x,
$$

where we used that $z(p)>\log p / \log \alpha$.

For the second part, since $z(p) \leq p+1 \leq 3 p / 2$, then

$$
Z_{\vartheta}(x)=\sum_{p \leq x} z(p) \ll \sum_{p \leq x} p \ll x \sum_{p \leq x} 1=x \pi(x) \ll \frac{x^{2}}{\log x}
$$

as desired.

\subsection{The Proof of Theorem 3}

Note that, by Theorem 2, we have

$$
Z_{\psi}(x) \geq Z_{\vartheta}(x) \gg x .
$$


For the second part, since there exist exactly $\lfloor\log x / \log p\rfloor$ powers of $p$ in the interval $[1, x]$, we can write $Z_{\psi}(x)$ as

$$
Z_{\psi}(x)=\sum_{p \leq x} \sum_{r \leq \log x} z\left(p^{r}\right)
$$

By using Lemma 1 (ii), we get

$$
Z_{\psi}(x)=\sum_{p \leq x} \sum_{r \leq \log x} z\left(p^{r}\right) \leq \sum_{p \leq x} \sum_{r \leq \log x}(p+1) p^{r-1} \ll \sum_{p \leq x} \sum_{r \leq \frac{\log x}{\log p}} p^{r} .
$$

Note that $\sum_{r \leq \frac{\log x}{\log p}} p^{r} \leq\left(p^{\frac{\log x}{\log p}+1}-1\right) /(p-1) \ll x$. Then,

$$
Z_{\psi}(x) \ll \sum_{p \leq x} x=x \pi(x) \ll \frac{x^{2}}{\log x}
$$

which completes the proof.

\section{Conclusions}

In this paper, we study some problems related to the order (of appearance) in the Fibonacci sequence, denoted by $z(n)$. This arithmetic function plays an important role in the comprehension of some Diophantine problems involving Fibonacci numbers (the most important one is the open problem about the existence of infinitely many Fibonacci prime numbers). The problems are related to the growth of Fibonacci versions of well-known number-theoretic functions (related to the Prime Number Theorem) like the first and second Chebyshev functions, $\vartheta(x)=\sum_{p \leq x} \log p$ and $\psi(x)=$ $\sum_{p^{r} \leq x} \log p$ and the harmonic function $H(x)=\sum_{n \leq x} 1 / n$. These Fibonacci-like functions are defined as $Z_{\vartheta}(x)=\sum_{p \leq x} z(p), Z_{\psi}(x)=\sum_{p^{r} \leq x} z\left(p^{r}\right)$ and $Z_{H}(x)=\sum_{n \leq x} z(n) / n$. In particular, we shall find effective bounds for these three functions. The proofs combine elementary facts related to $z(n)$ (such as Marques' upper bounds) together with some deep tools from Analytic Number Theory (such as Abel's summation formula and Sathé-Selberg formula).

Funding: The author was supported by Project of Excelence PrF UHK No. 2213/2020, University of Hradec Králové, Czech Republic.

Acknowledgments: The author thanks the anonymous referees for their careful corrections and very helpful and detailed comments, which have significantly improved the presentation of this paper.

Conflicts of Interest: The author declares no conflict of interest.

\section{References}

1. Trojovský, P. On Diophantine equations related to order of appearance in Fibonacci sequence. Mathematics 2019, 7, 1073. [CrossRef]

2. Lucas, E. Théorie des fonctions numériques simplement périodiques. Am. J. Math. 1878, 1, $289-321$. [CrossRef]

3. Sallé, H.J.A. Maximum value for the rank of apparition of integers in recursive sequences. Fibonacci Quart. 1975, 13, 159-161.

4. Marques, D. Sharper upper bounds for the order of appearance in the Fibonacci sequence. Fibonacci Quart. 2013, 51, 233-238.

5. Hast, D.R.; Matei, V. Higher Moments of Arithmetic Functions in Short Intervals: A Geometric Perspective. Int. Math. Res. Not. 2019, 21, 6554-6584. [CrossRef]

6. Visser, M. Primes and the Lambert W function. Mathematics 2018, 6, 56. [CrossRef]

7. Lagarias, J. An elementary problem equivalent to the Riemann hypothesis. Am. Math. Monthly 2002, 109, 534-543. [CrossRef] 
8. Sathé, L.G. On a problem of Hardy on the distribution of integers having a given number of prime factors. J. Indian Math. Soc. 1953, 17, 63-141.

9. Selberg, A.A. Note on a Paper by L.G. Sathe. J. Indian Math. Soc. 1954, 18, 83-87.

10. De Koninck, J.-M.; Luca, F. Analytic Number Theory-Exploring the Anatomy of Integers; American Mathematical Society: Providence, RI, USA, 2012.

(C) 2020 by the author. Licensee MDPI, Basel, Switzerland. This article is an open access article distributed under the terms and conditions of the Creative Commons Attribution (CC BY) license (http:/ / creativecommons.org/licenses/by/4.0/). 